\title{
Prognostic value of albumin to globulin ratio in non-muscle-invasive bladder cancer
}

\author{
Fahad Quhal ${ }^{1,2}$ (1) Benjamin Pradere ${ }^{1,3} \cdot$ Ekaterina Laukhtina $^{1,4} \cdot$ Reza Sari Motlagh $^{1} \cdot$ Hadi Mostafaei ${ }^{1,5} \cdot$ \\ Keiichiro Mori ${ }^{1,6} \cdot$ Victor M. Schuettfort ${ }^{1,7} \cdot$ Pierre I. Karakiewicz $^{8} \cdot$ Morgan Rouprêt $^{9} \cdot$ Dmitry Enikeev $^{4}$. \\ Michael Rink ${ }^{7}$. Mohammad Abufaraj ${ }^{10}$. Shahrokh F. Shariat ${ }^{1,4,10,11,12,13,14,15}$
}

Received: 13 September 2020 / Accepted: 29 December 2020 / Published online: 26 January 2021

(c) The Author(s) 2021

\begin{abstract}
Purpose To investigate the prognostic value of preoperative serum albumin to globulin ratio (AGR) in patients with nonmuscle-invasive bladder cancer (NMIBC) treated with transurethral resection of bladder tumor (TURB) with or without intravesical therapy (IVT).

Materials and methods We retrospectively reviewed 1,096 consecutive patients with NMIBC. Levels of albumin and globulin were obtained before TURB and used to calculate the preoperative AGR level. Multivariable Cox regression analyses were performed to assess the prognostic effect of preoperative AGR on oncologic outcomes. Subgroup analyses were performed in patients based on the European Association of Urology (EAU) risk groups for NMIBC.

Results Low AGR levels were observed in 389 (35.5\%) patients. The median follow-up was 63.7 months (IQR 25.3-111). On multivariable Cox regression analysis, low AGR was associated with increased risk of progression to muscle-invasive $\mathrm{BCa}$ (MIBC) (HR 1.81, 95\% CI 1.22-2.68, $P=0.003$ ). The addition of AGR only minimally improved the discrimination ability of a base model that included established clinicopathologic features $(\mathrm{C}$-index $=0.7354$ vs. C-index $=0.7162)$. Low preoperative AGR was not significantly associated with the risk of disease recurrence $(P=0.31)$. In subgroup analyses based on patients' EAU risk groups, low preoperative AGR was not associated with recurrence-free survival (RFS) $(P=0.59)$ or progression-free survival (PFS) $(P=0.22)$ in any of the risk groups. Additionally, in patients treated with Bacillus Calmette-Guerin (BCG) for intermediate- or high-risk NMIBC, low AGR failed to predict disease recurrence or progression. Conclusion Preoperative serum AGR levels independently predicted the risk of disease progression in patients with NMIBC. However, it was not found to be associated with either RFS or PFS in NMIBC patients based on their EAU risk group. This marker seems to have a limited role in NMIBC at the present time. However, further research is needed to investigate this marker in combination with other systemic inflammatory markers to help improve prediction in this heterogeneous group of patients.
\end{abstract}

Keywords Non-muscle-invasive · Bladder cancer · Progression · AGR · Albumin · Globulin · BCG

\section{Abbreviations}

AGR Albumin-to-globulin ratio

NMIBC Non-muscle-invasive bladder cancer

TURB Transurethral resection of bladder tumor

IVT Intravesical therapy

EAU The European Association of Urology

Supplementary Information The online version contains supplementary material available at https://doi.org/10.1007/s0034 5-020-03586-1.

Shahrokh F. Shariat

shahrokh.shariat@meduniwien.ac

Extended author information available on the last page of the article
MIBC Muscle-invasive BCa

BCG Bacillus Calmette-Guerin

$\mathrm{BCa} \quad$ Bladder cancer

RFS Recurrence-free survival

PF Progression free

\section{Introduction}

Bladder cancer (BCa) is the 9th most commonly diagnosed cancer, and the 13th cause of cancer-related mortality worldwide [1]. Approximately $70-80 \%$ of BCa patients present initially as non-muscle-invasive (NMIBC) [2]. The 
standard treatment for those patients is transurethral resection (TURB) followed by intravesical therapy (IVT) depending on the patient's risk. However, despite treatment, around $50 \%$ experience disease recurrence and about 10-20\% experience disease progression to muscle-invasive disease (MIBC) [2-4].

Patients with MIBC who experience disease progression from primary NMIBC have worse prognosis compared to those who present with primary MIBC, with up to $50 \%$ succumbing to their disease despite radical cystectomy $[5,6]$. Identifying those patients before disease progression is of utmost importance, as it may improve their outcomes by adapting treatment strategy [7-10]. The current risk prediction tools like the European Organization for Research and Treatment of Cancer (EORTC) or the Spanish Urological Club for Oncological Treatment (CUETO) scoring models rely on clinicopathologic features. While these prediction tools have improved the management of NMIBC, validation studies have reported their limited accuracy, especially in high-risk groups [4]. Identifying additional accurate biomarkers can potentially help improve the predictive ability of these models, to help tailor the treatment for those at higher risk of progression [11-15].

Albumin and globulin are major components of serum proteins. These proteins play an essential role in immunity and inflammation. Albumin-to-globulin ratio (AGR) has been reported to be a potential prognostic biomarker in several cancers $[16,17]$.

We hypothesized that low preoperative AGR is associated with worse oncologic outcomes in NMIBC patients. To test this theory, we studied the association between preoperative AGR and the risk of disease recurrence and progression in a large multicentric cohort of NMIBC patients. We also tested the prognostic value of preoperative AGR within each EAU risk group [2].

\section{Materials and methods}

\section{Patient population and treatment}

This study was approved by the institutional review boards of the participating centers. Waiver of individual informed consent was granted for this retrospective study. We retrospectively reviewed the medical records of 1,096 consecutive patients with NMIBC treated with TURB. Immediate singledose postoperative instillation chemotherapy, adjuvant intravesical chemotherapy, or adjuvant Bacillus Calmette-Guérin (BCG) immunotherapy were administered according to patient's risk group, guideline treatment recommendations and patient-physician shared decision-making. Repeat TURB was not routinely performed. All specimens were staged and graded according to the 2009 TNM classification and the 1973
World Health Organization system, respectively, by genitourinary pathologists. Serum albumin and globulin were collected within 30 days before TURB. No known systematic inflammatory disease or urinary tract infection was seen in all patients. Patients were assigned into low-, intermediate-, and high-risk groups of NMIBC, according to the European Association of Urology (EAU) guidelines [2].

\section{Follow-up}

The postoperative follow-up included physical examination, urine cytology, and cystoscopy scheduled generally at every 3 months for the first 2 years, every 6 months for the three following years, and then yearly. Imaging of the upper urinary tract was performed based on pathologic features according to guidelines and at physician discretion. Disease recurrence was defined as the first pathologically proven tumor relapse of any stage or grade, whereas disease progression was defined as muscle-invasive tumor [18].

\section{Finding the optimal cutoff value for preoperative AGR}

The preoperative AGR cut-off point was determined by Receiver Operating Characteristics (ROC) curve analysis using Youden index [19]. For the whole cohort, median preoperative AGR was 1.54 (1.38-1.69); the optimal cut-off value corresponding to the maximum joint sensitivity and specificity was 1.41. Based on this cutoff value, a total of 389 patients (35.5\%) were detected with a preoperative AGR $<1.41$ 'low preoperative AGR', whereas 797 patients (64.5\%) had an AGR $\geq 1.41$ 'normal preoperative AGR'.

\section{Statistical analysis}

Comparisons of study groups were assessed by Chi-square and Mann-Whitney $U$ tests. Kaplan-Meier curves and the logrank test were used to estimate and determine the statistical differences between study groups. Univariable and multivariable Cox regression analyses were used to test the association between preoperative AGR and oncologic outcomes. Exploratory subgroup analyses were performed in patients based on the EAU risk groups, and BCG-treated high-risk patients. Results were considered significant if two-sided $P$-value was $<0.05$. Data analyses were performed using STATA 16 (Stata Corp., College Station, TX). 


\section{Results}

\section{Association with clinical and pathologic features}

The clinicopathologic features of 1,096 patients with NMIBC treated with TURB are stratified by preoperative AGR level and summarized in Table 1. The median follow-up was 63.7 months (IQR 25.3-111). The median age for the overall cohort was 67 years (IQR 58-74). There were no statistically significant differences in age, gender, grade, stage, and tumor size or number between the low and normal preoperative AGR groups. There were more patients with concomitant carcinoma in-situ (CIS) in the low AGR group.

\section{Association with recurrence-free survival (RFS)}

During follow-up, 462 (42.2\%) patients developed pathologically confirmed disease recurrence, 177 (45.5\%) patients with low AGR and 285 (40.3\%) patients with normal AGR. The median time to recurrence was 27.5 months (IQR
Table 1 Clinicopathologic features of 1096 patients with non-muscle-invasive bladder cancer, stratified by preoperative serum albumin-to-globulin ratio (AGR)

\begin{tabular}{|c|c|c|c|c|}
\hline Variables & Total & Normal AGR & Low AGR & $P$-value \\
\hline Number of patients, $n(\%)$ & 1096 & 797 (64.5) & $389(35.5)$ & \\
\hline Median age (IQR) & $67(58-74)$ & $65.5(57.9-74)$ & $67.7(60-75)$ & 0.11 \\
\hline \multicolumn{5}{|l|}{ Gender, $n(\%)$} \\
\hline Female & $254(23.2)$ & $158(62.2)$ & $96(37.8)$ & \multirow[t]{2}{*}{0.38} \\
\hline Male & $842(76.8)$ & $549(65.2)$ & $293(34.8)$ & \\
\hline \multicolumn{5}{|l|}{ Smoking status, $n(\%)$} \\
\hline Never smoked & 267 & $160(59.93)$ & $107(40.1)$ & \multirow[t]{3}{*}{0.20} \\
\hline Former smoker & 322 & $213(66.2)$ & $109(33.8)$ & \\
\hline Current smoker & 507 & $334(65.9)$ & $173(34.1)$ & \\
\hline \multicolumn{5}{|l|}{ Prior recurrent rate, $n(\%)$} \\
\hline Primary & 916 & $600(65.5)$ & $316(34.5)$ & \multirow[t]{3}{*}{0.69} \\
\hline$\leq 1$ recurrent $/ y$ & 88 & $51(51.9)$ & $37(42.1)$ & \\
\hline$>1$ recurrent/y & 92 & $56(60.9)$ & $36(39.1)$ & \\
\hline \multicolumn{5}{|l|}{ Pathologic T stage, $n(\%)$} \\
\hline $\mathrm{pTa}$ & 653 & $419(64.2)$ & $234(35.8)$ & \multirow[t]{2}{*}{0.77} \\
\hline pT1 & 443 & $288(65)$ & $155(35)$ & \\
\hline \multicolumn{5}{|l|}{ Pathologic tumor grade, $n(\%)$} \\
\hline Grade 1 & 230 & $159(69.1)$ & $71(30.9)$ & \multirow[t]{3}{*}{0.21} \\
\hline Grade 2 & 383 & $238(62.1)$ & 145 (37.9) & \\
\hline Grade 3 & 483 & $310(64.2)$ & $173(35.8)$ & \\
\hline Concomitant CIS, $n(\%)$ & 47 & $23(48.9)$ & $24(51.1)$ & 0.023 \\
\hline \multicolumn{5}{|l|}{ Tumor size, $n(\%)$} \\
\hline$<1 \mathrm{~cm}$ & 352 & $235(66.8)$ & $117(33.2)$ & \multirow[t]{3}{*}{0.54} \\
\hline $1-3 \mathrm{~cm}$ & 444 & $280(63.1)$ & $164(36.9)$ & \\
\hline$>3 \mathrm{~cm}$ & 300 & $192(64)$ & $108(36)$ & \\
\hline \multicolumn{5}{|l|}{ Number of tumors, $n(\%)$} \\
\hline 1 & 704 & $453(64.35)$ & $251(35.65)$ & \multirow[t]{3}{*}{0.28} \\
\hline $2-7$ & 291 & $182(62.54)$ & $109(37.5)$ & \\
\hline$\geq 8$ & 101 & $72(71.3)$ & $29(28.7)$ & \\
\hline Intravesical therapy, $n(\%)$ & $472(43.1)$ & $294(62.3)$ & $178(37.7)$ & 0.18 \\
\hline \multicolumn{5}{|c|}{ Type of intravesical therapy, $n(\%)$} \\
\hline Early single instillation & 145 & $89(61.4)$ & $56(38.6)$ & \multirow[t]{3}{*}{0.60} \\
\hline Adjuvant chemotherapy & 48 & $30(62.5)$ & $18(37.5)$ & \\
\hline Adjuvant BCG & 279 & $175(62.72)$ & $104(37.28)$ & \\
\hline \multicolumn{5}{|l|}{ EAU risk group, $n(\%)$} \\
\hline Low-risk & 78 & $53(67.95)$ & $25(32.1)$ & \multirow[t]{3}{*}{0.76} \\
\hline Intermediate & 519 & $336(64.7)$ & $183(35.3)$ & \\
\hline High-risk & 499 & $318(63.7)$ & $181(36.3)$ & \\
\hline
\end{tabular}


8.3-68). Kaplan-Meier survival curves showed no significant difference in RFS between patients with low and normal serum AGR (supplementary Figure 1). There was no significant association between preoperative serum AGR and the risk of disease recurrence in univariable (HR 1.13, 95\% CI 0.94-1.37, $P=0.19$ ) and multivariable analyses (HR 1.10, 95\% CI 0.91-1.33, $P=0.31$ ) (Supplementary Table 1).

\section{Association with progression-free survival (PFS)}

During follow-up, 101 (9.2\%) patients experienced progression to MIBC; 52 (13.4\%) patients with low AGR and 49 (6.9\%) patients with normal AGR. The median time to progression was 25 months (IQR 8.8-68.3). Kaplan-Meier survival curves showed a significant difference in PFS between patients with low and normal serum AGR (Fig. 1). On a univariable analysis, low AGR was significantly associated with a higher risk of progression to MIBC (HR 1.99, 95\% CI 1.35-2.94, $P=0.001$ ). On multivariable analysis, preoperative AGR retained its independent association with PFS, after adjustment for the effects of established clinical and pathologic confounders (HR 1.81, 95\% CI 1.22-2.68, $P=0.003$ ). The inclusion of preoperative AGR marginally improved the discrimination of a base model that included established clinicopathologic features $(\mathrm{C}$-index $=0.7354$ vs. C-index $=0.7162)$ (Table 2).

\section{Subgroup analyses in patients based on their EAU risk group stratifications}

In 499 patients who were classified in the EAU high-risk group, $187(37.5 \%)$ and $62(12.4 \%)$ patients experienced disease recurrence and progression, respectively. The median follow-up was 57 months. There was no statistically

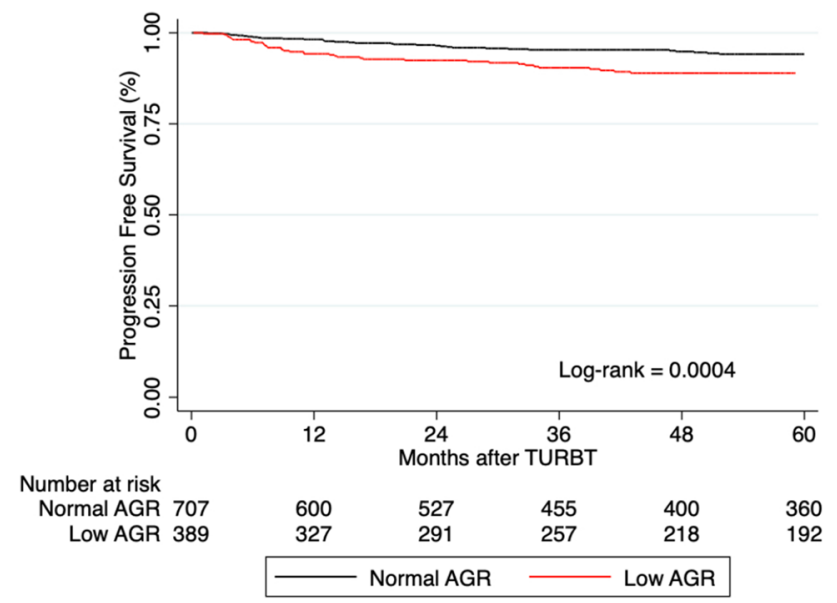

Fig. 1 Progression-free survival in 1096 patients with non-muscleinvasive bladder cancer, stratified by preoperative serum albumintoglobulin ratio (AGR) significant association between low AGR level and disease recurrence (HR 1.08 ; 95\% CI $0.81-1.46, P=0.59$ ) or progression (HR 1.38; 95\% CI 0.83-2.28, $P=0.22$ ). Furthermore, no significant associations were found with recurrence or progression in the EAU low-risk group patients (Supplementary Table 2).

\section{Subgroup analyses in patients according to intravesical BCG therapy}

Overall, 274 patients with EAU intermediate or high-risk group, who received adjuvant intravesical BCG therapy (induction and maintenance of at least 1 year). During a median follow-up of 59 months, $105(38.3 \%)$ patients experienced disease recurrence, and 21 (7.7\%) patients experienced disease progression to MIBC. Owing to the small sample size, the association with oncologic outcomes was tested in univariable analyses. Low preoperative AGR levels were not significantly associated with either disease recurrence (HR 1.12; 95\% CI 0.76-1.66, $P=0.57$ ) or progression (HR 1.65; 95\% CI 0.69-3.89, $P=0.26$ ) (Supplementary Table 2).

\section{Discussion}

In recent years, there has been an increased awareness of the role of inflammation in relation to BCa development, progression and metastasis [15, 20-26]. Several systemic inflammatory markers have been evaluated in the literature and showed promising results [27, 28]. However, none of these factors have sufficient level of evidence to be implemented in the evaluation and management of $\mathrm{BCa}[11,29]$. The current prognostic tools for patients with NMIBC rely mainly on pathologic features of the tumor [30, 31]; integrating novel biomarkers can help improve the prognostic ability of these models $[12,13]$. In a retrospective study of three systemic inflammatory markers (neutrophil-to-lymphocyte ratio (NLR), platelet-to-lymphocyte ratio (PLR), and lymphocyte-to-monocyte ratio (LMR), Cantiello et al. found that their combination in a predictive multivariable model can predict the risk of disease recurrence and progression in patients with high-risk NMIBC [22]. Similarly, D’Andrea et al. found that preoperative neutrophil-to-lymphocyte ratio is associated with both RFS and PFS in patients with NMIBC [20]. However, no previous study formally tested whether addition of AGR improves discrimination ability.

In this study, we investigated the association between preoperative AGR and NMIBC. We found that low preoperative AGR level predicts the risk of disease progression to MIBC. However, it did not predict the risk of disease recurrence. Only one study has previously evaluated the association of AGR with NMIBC. Niwa et al. found that low AGR was 
Table 2 Univariable and multivariable Cox regression analyses for the prediction of progression-free survival in 1,096 patients with non-muscleinvasive bladder cancer

\begin{tabular}{|c|c|c|c|c|c|c|}
\hline \multicolumn{7}{|c|}{ Progression free survival } \\
\hline \multirow[t]{2}{*}{ Variable } & \multicolumn{3}{|l|}{ Univariable } & \multicolumn{3}{|c|}{ Multivariable } \\
\hline & HR & $95 \% \mathrm{CI}$ & $p$-value & $\overline{\mathrm{HR}}$ & $95 \% \mathrm{CI}$ & $p$-value \\
\hline Age & 1.04 & $1.02-1.06$ & $<0.001$ & 1.04 & $1.02-1.01$ & $<0.001$ \\
\hline \multicolumn{7}{|l|}{ Gender } \\
\hline Male & Reference & Reference & Reference & & & \\
\hline Female & 1.20 & $0.77-1.86$ & 0.42 & & & \\
\hline \multicolumn{7}{|l|}{ Pathologic T stage } \\
\hline pTa & Reference & Reference & Reference & Reference & Reference & Reference \\
\hline pT1 & 1.57 & $1.06-2.32$ & 0.02 & 0.48 & $0.23-0.99$ & 0.050 \\
\hline \multicolumn{7}{|l|}{ Tumor grade } \\
\hline G1 & Reference & Reference & Reference & Reference & Reference & Reference \\
\hline G2 & 2.56 & $1.19-5.52$ & 0.02 & 2.14 & $0.98-4.66$ & 0.057 \\
\hline G3 & 3.92 & $1.87-8.22$ & $<0.001$ & 6.04 & $2.21-16.50$ & $<0.001$ \\
\hline Concomitant CIS & 1.37 & $0.52-3.38$ & 0.49 & 0.79 & $0.31-1.99$ & 0.61 \\
\hline \multicolumn{7}{|l|}{ Tumor size } \\
\hline$<1 \mathrm{~cm}$ & Reference & Reference & Reference & Reference & Reference & Reference \\
\hline $1-3 \mathrm{~cm}$ & 1.53 & $0.92-2.53$ & 0.10 & 1.38 & $0.82-2.31$ & 0.22 \\
\hline$>3 \mathrm{~cm}$ & 1.80 & $1.08-3.01$ & 0.025 & 1.40 & $0.82-2.37$ & 0.22 \\
\hline \multicolumn{7}{|l|}{ Number of tumors } \\
\hline Single & Reference & Reference & Reference & Reference & Reference & Reference \\
\hline $2-7$ & 1.50 & $0.96-2.35$ & 0.08 & 1.23 & $0.78-1.95$ & 0.38 \\
\hline$\geq 8$ & 2.61 & $1.49-4.55$ & 0.001 & 2.19 & $1.24-3.88$ & 0.007 \\
\hline Intravesical therapy & 1.13 & $0.76-1.68$ & 0.55 & 0.94 & $0.61-1.45$ & 0.78 \\
\hline \multicolumn{7}{|l|}{ AGR } \\
\hline Normal & Reference & Reference & Reference & Reference & Reference & Reference \\
\hline Low & 1.99 & $1.35-2.94$ & 0.001 & 1.81 & $1.22-2.68$ & 0.003 \\
\hline
\end{tabular}

associated with a higher risk of both disease recurrence and progression in 364 patients with primary NMIBC [32]. In our study, we tested this lead further within a larger cohort of patients. We also performed subgroup analyses to identify the group of patients in whom this biomarker would likely be of most clinical benefit.

Patients with high-risk NMIBC are known to be subject to misclassification based on the current risk stratification tools. Risk assessment models for NMIBC, such as the EORTC and CUETO, have been criticized for the overestimation of the risk of disease progression, especially in patients with high-risk NMIBC [4, 33, 34]. In our study, we tested preoperative AGR in subgroups of patients based on their EAU risk groups; however, this biomarker failed to predict disease recurrence and progression in any of the risk groups. The reason underlying this finding can be multifold, such as the statistical power and the short follow-up.

Management of BCG unresponsive BCa remains one of the most challenging problems in urology, as these tumors have the highest risk of disease progression as well as metastasis [35-37]. Unfortunately, no current biomarker has sufficient evidence to identify the patients who are most likely to fail BCG therapy, which leads to delay in potential curative therapies (e.g., radical cystectomy) [37-39]. In our study, we further examined this biomarker in the subgroup of intermediate- and high-risk patients who were treated with BCG; here as well, there was no statistical association of preoperative AGR with disease recurrence or progression.

Albumin and globulin are major serum proteins and can reflect the systemic inflammatory response. Albumin modulates the systemic inflammatory reaction, as well as exert antioxidant effects. Albumin also plays an essential role in stabilizing cell growth and DNA replication. A low level of albumin has been a useful prognostic tool for various cancers. Globulin, on the other hand, increases with the accumulation of acute-phase proteins and immunoglobulins, which are reflective of an immunologic and inflammatory state [40, 41]. Emerging evidence has shown that AGR can be a useful predictive tool for cancer diagnosis and prognosis. Indeed, several studies reported that low serum AGR is correlated with worse outcomes in several cancers, such as gastric [16], colorectal [42], lung [43], and breast [44] cancers. However, this biomarker seems to have limited prognostic benefit in patients with NMIBC. Nevertheless, its 
association in our study with the risk of disease progression within the whole cohort can hint for a possible prognostic benefit. Probably further studies with better design (larger numbers, longer follow-up and more events) can evaluate this biomarker either alone, or in combination with other systemic inflammatory markers in this setting of $\mathrm{BCa}$ or, even, as a predictor to response of systemic therapies, such as immune checkpoint blockade.

The present study has several limitations, first, the retrospective design with its inherent selection bias. Second, because this is a multicentric study, different surgeons performed the TURB and the specimens were not reviewed by a central pathology. However, all participating centers are high-volume centers with experienced surgeons and genitourinary pathologists. Moreover, due to the multicentric nature of the study, repeat TURB was not routinely performed. Despite these limitations, we were able to provide a descriptive analysis of this biomarker in the different groups of NMIBC patients.

\section{Conclusion}

Systemic inflammatory markers have a promising role in cancer prognosis. Low preoperative AGR was associated with a higher risk of disease progression in NMIBC patients but not with disease recurrence. Nevertheless, it failed to predict the risk of either recurrence or progression in patients based on their EAU risk groups and in BCG-treated patients. Further studies are needed to evaluate this marker either in its own or in combination with other systemic inflammatory markers to help build an optimal prognostic model for NMIBC and to guide therapeutic decision-making.

\begin{abstract}
Author contributions FQ: Protocol/project development, Data analysis, Manuscript writing/editing, BP: Data analysis, Manuscript writing/ editing, EL: Data analysis, Manuscript writing/editing, RS: Manuscript writing/editing, HM: Manuscript writing/editing, KM: Data analysis, Manuscript writing/editing, VS: Manuscript writing/editing, PI.K: Manuscript writing/editing, MR: Manuscript writing/editing, DE: Manuscript writing/editing, MR: Manuscript writing/editing, MA: Manuscript writing/editing, SF.S: Protocol/project development, Data collection or management, Manuscript writing/editing.
\end{abstract}

Funding Open Access funding provided by Medical University of Vienna.

\section{Compliance with ethical standards}

Conflict of interest The authors declare that they have no conflict of interest.
Research involving Human participants and/or animals This article does not contain any studies with animals performed by any of the authors.

Informed consent Informed consent was obtained from all individual participants included in the study.

Open Access This article is licensed under a Creative Commons Attribution 4.0 International License, which permits use, sharing, adaptation, distribution and reproduction in any medium or format, as long as you give appropriate credit to the original author(s) and the source, provide a link to the Creative Commons licence, and indicate if changes were made. The images or other third party material in this article are included in the article's Creative Commons licence, unless indicated otherwise in a credit line to the material. If material is not included in the article's Creative Commons licence and your intended use is not permitted by statutory regulation or exceeds the permitted use, you will need to obtain permission directly from the copyright holder. To view a copy of this licence, visit http://creativecommons.org/licenses/by/4.0/.

\section{References}

1. Antoni S, Ferlay J, Soerjomataram I et al (2017) Bladder cancer incidence and mortality: a global overview and recent trends. Eur Urol 71:96

2. Babjuk M, Burger M, Comperat EM et al (2019) European Association of Urology Guidelines on non-muscle-invasive bladder cancer (TaT1 and carcinoma in situ) - 2019 Update. Eur Urol 76:639

3. Gontero P, Sylvester R, Pisano F et al (2015) Prognostic factors and risk groups in T1G3 non-muscle-invasive bladder cancer patients initially treated with Bacillus Calmette-Guérin: results of a retrospective multicenter study of 2451 patients. Eur Urol 67:74

4. Xylinas E, Kent M, Kluth L et al (2013) Accuracy of the EORTC risk tables and of the CUETO scoring model to predict outcomes in non-muscle-invasive urothelial carcinoma of the bladder. Br J Cancer 109:1460

5. Shariat SF, Karakiewicz PI, Palapattu GS et al (2006) Outcomes of radical cystectomy for transitional cell carcinoma of the bladder: a contemporary series from the Bladder Cancer Research Consortium. J Urol 176:2414

6. Schrier BP, Hollander MP, van Rhijn BW et al (2004) Prognosis of muscle-invasive bladder cancer: difference between primary and progressive tumours and implications for therapy. Eur Urol 45:292

7. Abufaraj M, Foerster B, Schernhammer E et al (2019) Micropapillary urothelial carcinoma of the bladder: a systematic review and meta-analysis of disease characteristics and treatment outcomes. Eur Urol 75:649

8. Mari A, Kimura S, Foerster B et al (2019) A systematic review and meta-analysis of the impact of lymphovascular invasion in bladder cancer transurethral resection specimens. BJU Int 123:11

9. Fritsche HM, Burger M, Svatek RS et al (2010) Characteristics and outcomes of patients with clinical T1 grade 3 urothelial carcinoma treated with radical cystectomy: results from an international cohort. Eur Urol 57:300

10. Svatek RS, Shariat SF, Novara G et al (2011) Discrepancy between clinical and pathological stage: external validation of the impact on prognosis in an international radical cystectomy cohort. BJU Int 107:898

11. Shariat SF, Lotan Y, Vickers A et al (2010) Statistical consideration for clinical biomarker research in bladder cancer. Urol Oncol 28:389 
12. Soria F, Krabbe LM, Todenhöfer T et al (2019) Molecular markers in bladder cancer. World J Urol 37:31

13. Schmitz-Dräger BJ, Droller M, Lokeshwar VB et al (2015) Molecular markers for bladder cancer screening, early diagnosis, and surveillance: the WHO/ICUD consensus. Urol Int 94:1

14. Shariat SF, Kim J, Raptidis G et al (2003) Association of p53 and p21 expression with clinical outcome in patients with carcinoma in situ of the urinary bladder. Urology 61:1140

15. Shariat SF, Kim JH, Ayala GE et al (2003) Cyclooxygenase-2 is highly expressed in carcinoma in situ and $\mathrm{T} 1$ transitional cell carcinoma of the bladder. J Urol 169:938

16. Mao MJ, Wei XL, Sheng H et al (2017) Clinical significance of preoperative albumin and globulin ratio in patients with gastric cancer undergoing treatment. Biomed Res Int 2017:3083267

17. Li J., Wang Y., Wu Y. et al. Prognostic value of pretreatment albumin to globulin ratio in lung cancer: a meta-analysis. Nutr Cancer: 1,2020

18. Kimura S, Soria F et al (2019) Prognostic value of modified glasgow prognostic score in non-muscle-invasive bladder cancer. Urol Oncol 37:179.e19

19. Youden WJ (1950) Index for rating diagnostic tests. Cancer 3:32

20. D'Andrea D, Moschini M, Gust K et al (2017) Prognostic role of neutrophil-to-lymphocyte ratio in primary non-muscle-invasive bladder cancer. Clin Genitourin Cancer 15:e755

21. Lucca I, Jichlinski P, Shariat SF et al (2016) The neutrophil-tolymphocyte ratio as a prognostic factor for patients with urothelial carcinoma of the bladder following radical cystectomy: validation and meta-analysis. Eur Urol Focus 2:79

22. Cantiello F, Russo GI, Vartolomei MD et al (2018) Systemic inflammatory markers and oncologic outcomes in patients with high-risk non-muscle-invasive urothelial bladder cancer. Eur Urol Oncol 1:403

23. Lucca I, Hofbauer SL, Leitner CV et al (2016) Development of a preoperative nomogram incorporating biomarkers of systemic inflammatory response to predict nonorgan-confined urothelial carcinoma of the bladder at radical cystectomy. Urology 95:132

24. Xylinas E, Robinson BD, Kluth LA et al (2014) Association of T-cell co-regulatory protein expression with clinical outcomes following radical cystectomy for urothelial carcinoma of the bladder. Eur J Surg Oncol 40:121

25. Shariat SF, Matsumoto K, Kim J et al (2003) Correlation of cyclooxygenase- 2 expression with molecular markers, pathological features and clinical outcome of transitional cell carcinoma of the bladder. J Urol 170:985

26. Shariat SF, Bolenz C, Godoy G et al (2009) Predictive value of combined immunohistochemical markers in patients with pT1 urothelial carcinoma at radical cystectomy. J Urol 182:78

27. Shariat SF, Kim JH, Andrews B et al (2001) Preoperative plasma levels of transforming growth factor beta(1) strongly predict clinical outcome in patients with bladder carcinoma. Cancer 92:2985

28. Andrews B, Shariat SF, Kim JH et al (2002) Preoperative plasma levels of interleukin-6 and its soluble receptor predict disease recurrence and survival of patients with bladder cancer. J Urol $167: 1475$

29. Bensalah K, Montorsi F, Shariat SF (2007) Challenges of cancer biomarker profiling. Eur Urol 52:1601
30. Sylvester RJ, van der Meijden AP, Oosterlinck W et al (2006) Predicting recurrence and progression in individual patients with stage Ta T1 bladder cancer using EORTC risk tables: a combined analysis of 2596 patients from seven EORTC trials. Eur Urol 49:466

31. Fernandez-Gomez J, Madero R, Solsona E et al (2009) Predicting nonmuscle invasive bladder cancer recurrence and progression in patients treated with Bacillus Calmette-Guerin: the CUETO scoring model. J Urol 182:2195

32. Niwa N, Matsumoto K, Ide $\mathrm{H}$ et al (2018) Prognostic value of pretreatment albumin-to-globulin ratio in patients with non-muscleinvasive bladder cancer. Clin Genitourin Cancer 16:e655

33. Rieken M, Shariat SF, Kluth L et al (2018) Comparison of the EORTC tables and the EAU categories for risk stratification of patients with nonmuscle-invasive bladder cancer. Urol Oncol 36:8.e17

34. Fernandez-Gomez J, Madero R, Solsona E et al (2011) The EORTC tables overestimate the risk of recurrence and progression in patients with non-muscle-invasive bladder cancer treated with Bacillus Calmette-Guérin: external validation of the EORTC risk tables. Eur Urol 60:423

35. Lerner SP, Tangen CM, Sucharew $\mathrm{H}$ et al (2009) Failure to achieve a complete response to induction BCG therapy is associated with increased risk of disease worsening and death in patients with high risk non-muscle invasive bladder cancer. Urol Oncol 27:155

36. Kamat AM, Sylvester RJ, Bohle A et al (2016) Definitions, end points, and clinical trial designs for non-muscle-invasive bladder cancer: recommendations from the International Bladder Cancer Group. J Clin Oncol 34:1935

37. Hassler MR, Shariat SF, Soria F (2019) Salvage therapeutic strategies for Bacillus Calmette-Guerin failure. Curr Opin Urol 29:239

38. Kamat AM, Li R, O'Donnell MA et al (2018) Predicting response to intravesical bacillus calmette-guérin immunotherapy: are we there yet? Sys Rev Eur Urol 73:738

39. Shariat SF, Enikeev DV, Mostafaei H (2019) Six essential conditions for bladder-sparing strategies in bacillus Calmette-Guérin unresponsive bladder cancer. Immunotherapy 11:1083

40. Balkwill FR, Mantovani A (2012) Cancer-related inflammation: common themes and therapeutic opportunities. Semin Cancer Biol $22: 33$

41. Rasouli M, Okhovatian A, Enderami A (2005) Serum proteins profile as an indicator of malignancy: multivariate logistic regression and ROC analyses. Clin Chem Lab Med 43:913

42. Azab B, Kedia S, Shah N et al (2013) The value of the pretreatment albumin/globulin ratio in predicting the long-term survival in colorectal cancer. Int J Colorectal Dis 28:1629

43. Duran AO, Inanc M, Karaca H et al (2014) Albumin-globulin ratio for prediction of long-term mortality in lung adenocarcinoma patients. Asian Pac J Cancer Prev 15:6449

44. Azab BN, Bhatt VR, Vonfrolio S et al (2013) Value of the pretreatment albumin to globulin ratio in predicting long-term mortality in breast cancer patients. Am J Surg 206:764

Publisher's Note Springer Nature remains neutral with regard to jurisdictional claims in published maps and institutional affiliations. 


\section{Authors and Affiliations}

\section{Fahad Quhal ${ }^{1,2}$ (D) Benjamin Pradere ${ }^{1,3} \cdot$ Ekaterina Laukhtina $^{1,4} \cdot$ Reza Sari Motlagh $^{1} \cdot$ Hadi Mostafaei $^{1,5}$. Keiichiro Mori $^{1,6} \cdot$ Victor M. Schuettfort $^{1,7} \cdot$ Pierre I. Karakiewicz $^{8} \cdot$ Morgan Rouprêt $^{9} \cdot$ Dmitry Enikeev $^{4}$. Michael Rink ${ }^{7}$. Mohammad Abufaraj ${ }^{10}$ • Shahrokh F. Shariat ${ }^{1,4,10,11,12,13,14,15}$}

1 Department of Urology, Comprehensive Cancer Center, Medical University of Vienna, Vienna, Austria

2 Department of Urology, King Fahad Specialist Hospital, Dammam, Saudi Arabia

3 Department of Urology, University Hospital of Tours, Tours, France

4 Institute for Urology and Reproductive Health, Sechenov University, Moscow, Russia

5 Research Center for Evidence Based Medicine, Tabriz University of Medical Sciences, Tabriz, Iran

6 Department of Urology, Jikei University School of Medicine, Tokyo, Japan

7 Department of Urology, University Medical Center Hamburg-Eppendorf, Hamburg, Germany

8 Cancer Prognostics and Health Outcomes Unit, Division of Urology, University of Montreal Health Center, Montreal, Canada
9 Predictive onco-uro, AP-HP, Urology Hôpital Pitié-Salpêtrière, Sorbonne Université, GRC n5, 75013 Paris, France

10 Division of Urology, Department of Special Surgery, Jordan University Hospital, The University of Jordan, Amman, Jordan

11 Department of Urology, Weill Cornell Medical College, New York, NY, USA

12 Department of Urology, University of Texas Southwestern, Dallas, TX, USA

13 Department of Urology, Second Faculty of Medicine, Charles University, Prague, Czech Republic

14 European Association of Urology Research Foundation, Arnhem, Netherlands

15 Karl Landsteiner Institute, Wahringer Gurtel 18-20, 1090 Vienna, Austria 